\title{
Scattering of Light by Photonic Crystals
}

\author{
B.J. Hoenders, M. Doosje* and J. Knoester \\ Institute for Theoretical Physics and Materials Science Centre \\ University of Groningen \\ Nijenborgh 4, 9747 AG Groningen, The Netherlands
}

\begin{abstract}
A new method is developed to calculate the scattering of light at the surface of a photonic crystal. The problem is solved in terms of virtual surface-current distributions and the calculation takes full advantage of the infinite-space plane-wave expansion method for obtaining the photonic band structure. Working with surface currents makes the calculations less time-consuming by means of reduction of the dimensionality in the problem. The method is applied to a semi-infinite dielectric continuum and for semi-infinite two-dimensional photonic crystals of small and large dielectric contrast.
\end{abstract}

PACS numbers: 42.70.Qs, 42.25.Fx, 42.25.Bs, 03.50.De, 41.20.Jb

\section{Introduction}

Photonic crystals are materials with a spatially periodic dielectric function $\varepsilon(\boldsymbol{r})$. Their dispersion relation $\omega(\boldsymbol{k})$ is described by the photonic band structure, which consists of different photonic bands, showing gaps between them at the boundary of the Brillouin zone (BZ). The width of a photonic band gap depends strongly on the dielectric contrast and on the geometrical configuration of the crystal structure. The interest in these materials started with the pioneering work of Bykov [1], Yablonovitch [2], and John [3] on the localization of light and the inhibition of spontaneous emission. Research activities on photonic crystals and photonic band structures have increased enormously since that time [4-7].

One of the main motivations to produce dielectric structures possessing large photonic band gaps, is the desired ability to control the spontaneous emission of radiation from atoms $[1-3,8]$.

*corresponding author; e-mail: B.J.Hoenders@phys.rug.nl 
Let us suppose that we have a photonic crystal with an omnidirectional photonic band gap for a frequency range $\omega_{1}<\omega<\omega_{2}$. If an atom, which naturally emits radiation of a frequency which lies inside this range, is placed inside the photonic crystal, then it cannot emit its radiation, because electromagnetic waves of that particular frequency cannot propagate through the crystal. This is the principle used in e.g. the measurement of fluorescence spectra of dye incorporated in photonic crystals, in experiments aimed at the detection of photonic band gaps $[9,10]$.

Another example is the suppression of molecular interactions in periodic dielectric structures [11]. Control of spontaneous emission is relevant for lowering the laser threshold in semiconductor lasers [2]. Another application for this effect is a single-mode light-emitting diode, proposed by Yablonovitch $[12,13]$.

Other possibilities for practical applications of photonic crystals include the realization of low-threshold lasers, single-mode lossless waveguides. The principle for photonic-crystal waveguides [14-23] is to guide light with a frequency inside the band gap of the photonic crystal along lattice defects which provide localised modes for this frequency. The lattice defects can be rows of point defects, but also linear or planar defects. Special features such as sharply bent waveguides [15-18] and branched waveguide structures ("beam splitters") [18-20] have also been developed.

Further applications include: high-quality resonant cavities [24, 25], perfectly reflecting mirrors and substrates for antennas [26, 27]. See also [28] for the calculation of the largest width of the band gap of a $3 \mathrm{D} \mathrm{fcc}$ air-sphere crystal optimizing the values of the pertinent geometrical parameters.

During the past decade, much theoretical effort in this field has been devoted to calculating photonic band structures and densities of states for ideal infinite crystals [4, 28-32]. However, such calculations do not account for the scattering of the light at the crystal's surface. This scattering problem has received relatively little attention. Methods that have been developed, include the generalised field-propagator method [33] and the transfer-matrix method [34, 35]. The former formulates the scattering problem in terms of three-dimensional vector integral equations, whose numerical solution is a time-consuming process. The latter assumes that the crystal can be built up from thin infinite layers and has such disadvantages that it is restricted to slab structures and excitation by plane-wave fields.

We present a rigorous alternative method, which is generally applicable to two- and three-dimensional crystals of arbitrary shape and to incident monochromatic fields of arbitrary spatial form. Moreover, the method utilises as input the infinite-space photonic band structure, which can be calculated by well-known methods. The advantage of the latter is that, once calculated for a particular material, this band structure may be used to calculate the scattering for any crystal shape and incident field. 


\section{Theory}

We consider the scattering of monochromatic electromagnetic waves of frequency $\omega$ at the surface of a photonic crystal. Our solution of this problem is based on the general property that the solutions to Maxwell's equations in a medium with a boundary surface $S$, may be considered as arising from equivalent tangential (virtual) surface-current distributions just above $S$. Thus, the magnetic components $\boldsymbol{H}(r, t)$ of the electromagnetic fields outside (inside) $S$ may be expressed in terms of integrals over the surface-current distribution $\boldsymbol{J}_{1}\left(\boldsymbol{J}_{2}\right)$, using an appropriate Green tensor (Fig. 1).

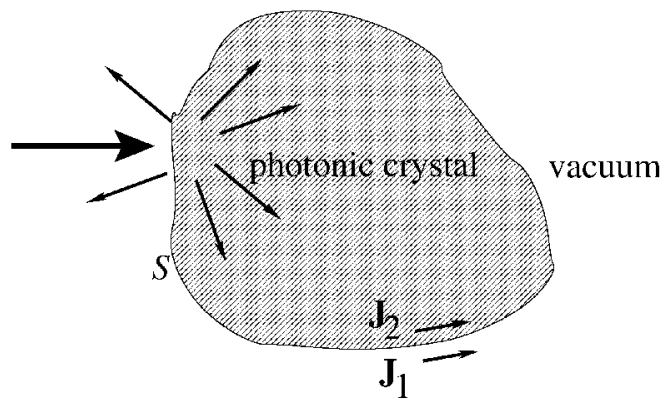

Fig. 1. Scattering of light by a photonic crystal. The electromagnetic fields outside the crystal are generated by the virtual current $\boldsymbol{J}_{1}$ on the outside of the boundary surface $S$. The fields inside the crystal are generated by $\boldsymbol{J}_{2}$.

The crux of our method is that one is rather flexible in the choice of this Green tensor. It should only obey Eq. (3) inside the medium of interest, but, except for satisfying the radiation condition at infinity, no particular boundary conditions are to be imposed on it. This allows us to work with infinite-space Green's tensors, even though we are interested in finite media. While this may appear paradoxal, we point out that for the scalar wave equation this is well known [36]; the extension to the vector wave equation can be proved in a very analogous way.

The result being that any solution of the scalar Helmholtz- or potential equation in a finite or semi-infinite volume $V$ can be written as the superposition of the fields generated by source- or dipole distributions located at the boundary of the volume $V$. In fact, our theory is a generalization of Huygens' principle for electromagnetic waves: the field is supposed to be generated by a weighted superposition of the fields arising from e.g. source- or dipole distributions located at an appropriate boundary.

Having expressed the fields in terms of the virtual surface currents, the scattering problem is solved by imposing the continuity of the tangential components of the magnetic and the electric fields across $S$. This leads to a set of linear equations 
for the, hitherto unknown, surface currents. Solution of these equations suffices to obtain the fields everywhere. We note that for a $d$-dimensional photonic crystal, the equations for the surface currents have a dimensionality of only $d-1$, which is an important computational advantage of our method.

We now turn to the explicit formulation of the solution. Omitting the time-dependence $\mathrm{e}^{\mathrm{i} \omega t}$, the magnetic field on the vacuum side of the interface $S$, reads

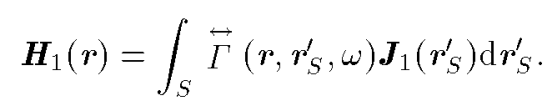

Here $\overleftrightarrow{\Gamma}$ is the dyadic Green tensor for electromagnetic waves in vacuum, which depends on the dimensionality of the problem (see below). Similarly, on the crystal side of the interface $S$, the magnetic field is expressed as

$$
\boldsymbol{H}_{2}(\boldsymbol{r})=\int_{S} \overleftrightarrow{\mathcal{G}}\left(r, \boldsymbol{r}_{S}^{\prime}, \omega\right) \boldsymbol{J}_{2}\left(\boldsymbol{r}_{S}^{\prime}\right) \mathrm{d} \boldsymbol{r}_{S}^{\prime}
$$

where $\overrightarrow{\mathcal{G}}$ is the dyadic Green tensor for the infinite photonic crystal satisfying the inhomogeneous wave equation

$$
\nabla \times\left[\frac{1}{\varepsilon(r)} \nabla \times \overleftrightarrow{\mathcal{G}}\left(r, r_{S}^{\prime}, \omega\right)\right]-(\omega / c)^{2} \stackrel{\leftrightarrow}{\mathcal{G}}\left(r, r_{S}^{\prime}, \omega\right)=\overrightarrow{\mathcal{I}} \delta\left(r-r_{S}^{\prime}\right)
$$

We would like to note once again the essential point that this particular choice for the Green tensor is certainly not unique, but that any solution of Eq. (3) valid inside $V$ could be used!

The dyadic Green tensor for the infinite photonic crystal reads

$$
\overrightarrow{\mathcal{G}}\left(\boldsymbol{r}, \boldsymbol{r}_{S}^{\prime}, \omega\right)=\sum_{n} \int_{1 \mathrm{BZ}} \frac{\tilde{\boldsymbol{h}}_{n}(\boldsymbol{r} ; \boldsymbol{k}) \otimes \tilde{\boldsymbol{h}}_{n}^{*}\left(\boldsymbol{r}_{S}^{\prime} ; \boldsymbol{k}\right)}{\left[\left(\omega_{n}(\boldsymbol{k})\right)^{2}-\omega^{2}+\gamma^{2}+2 \mathrm{i} \gamma \omega\right] / c^{2}} \mathrm{~d} \boldsymbol{k} .
$$

Here $\tilde{\boldsymbol{h}}_{n}(\boldsymbol{r} ; \boldsymbol{k})$ are the normalised Bloch modes

$$
\tilde{\boldsymbol{h}}_{n}(\boldsymbol{r} ; \boldsymbol{k}) \equiv \sum_{m} \sum_{\lambda=1}^{2} \tilde{h}_{m \lambda}^{(n)}(\boldsymbol{k}) \hat{\boldsymbol{u}}_{m}^{\lambda} \mathrm{e}^{-\mathrm{i} \boldsymbol{k}_{m} \cdot \boldsymbol{r}},
$$

defined uniquely by the equation

$$
\nabla \times\left[\frac{1}{\varepsilon(r)} \nabla \times \tilde{h}_{n}(r ; \boldsymbol{k})\right]=\frac{\omega_{n}^{2}}{c^{2}} \tilde{h}_{n}(r ; \boldsymbol{k})
$$

and the condition that the fields are bounded at $|r| \rightarrow \infty$. The index $n$ is used for labelling the eigenfrequencies $\omega_{n}$ and the eigenvectors $\tilde{h}_{m \lambda}^{(n)}(\boldsymbol{k})$. These Bloch modes are the eigenvectors, which are calculated in the standard plane-wave expansion method, that is used to obtain the photonic band structure [4, 29]. By $\hat{\boldsymbol{u}}_{m}^{\lambda}, \lambda=$ $1,2,3$, we denote an orthonormal basis for Euclidean space, for each index $m$ which labels a reciprocal-lattice vector $\boldsymbol{g}_{m}$. The vector $\boldsymbol{k}_{m}$ equals $\boldsymbol{k}+\boldsymbol{g}_{m}$. Our definition for $-G$ is the same as in Ref. [37], except for the fact that we do not need to 
incorporate the longitudinal Bloch modes due to $\nabla \cdot \boldsymbol{H}=0$. The small absorption parameter $\gamma$ has been introduced to handle the singularity of the Green tensor along the dispersion curves in $k$-space where $\omega_{n}(\boldsymbol{k})=\omega$.

Using the above representation of the fields on either side of $S$, the unknown surface-current distributions $\boldsymbol{J}_{1}$ and $\boldsymbol{J}_{2}$ can be solved by requiring continuity of the tangential components of $\boldsymbol{H}$ (i.e. continuity of $n \times \boldsymbol{H}$ ) and the tangential components of $\boldsymbol{E}$ (i.e. continuity of $\boldsymbol{n} \times \boldsymbol{E}$, or, equivalently, of $\boldsymbol{n} \times(\nabla \times \boldsymbol{H}) / \varepsilon(r)$ ) [38]. Here, $\boldsymbol{n}$ is the unit vector perpendicular to $S$. In practice, imposing the boundary conditions leads to a linear set of equations for the discretised surface-current distributions. Once these distributions have been solved, they may be substituted back into Eqs. (1) and (2) to calculate the fields anywhere. In particular we are interested in the far field distributions of the scattered field.

Thus, in practice our method involves:

a) the construction of the Green tensor of the photonic crystal using standard band-structure calculations,

b) the solution of a set of linear equations for the discretised surface-current distributions, and

c) the calculation of the fields of interest using the solution obtained for the currents.

\section{Calculation of the photonic band-structure: the plane-wave expansion method \\ 3.1. Maxwell's equations and the electromagnetic wave equation}

In any physical situation where electric and/or magnetic fields play a role, these fields must be a solution of Maxwell's equations. The next step is usually to derive the wave equation from Maxwell's equations.

Thus, let us start with Maxwell's equations in SI units, in the presence of free charges and currents

$$
\begin{array}{ll}
\nabla \cdot \boldsymbol{D}=\rho, & \nabla \times \boldsymbol{E}=-\frac{\partial \boldsymbol{B}}{\partial t}, \\
\nabla \cdot \boldsymbol{B}=0, & \nabla \times \boldsymbol{H}=\frac{\partial \boldsymbol{D}}{\partial t}+\boldsymbol{J},
\end{array}
$$

where the displacement field $\boldsymbol{D}=\varepsilon_{0} \boldsymbol{E}+\boldsymbol{P}$ and the magnetic field $\boldsymbol{B}=\mu_{0} \boldsymbol{H}+\boldsymbol{M}$. Without the free charges and currents, these equations are written as

$$
\begin{array}{ll}
\nabla \cdot \boldsymbol{D}=0, & \nabla \times \boldsymbol{E}=-\frac{\partial \boldsymbol{B}}{\partial t}, \\
\nabla \cdot \boldsymbol{B}=0, & \nabla \times \boldsymbol{H}=\frac{\partial \boldsymbol{D}}{\partial t} .
\end{array}
$$

In these equations we did not specify the dependence of the fields on the space coordinate $r$ and on the time $t$ explicitly, but it should be obvious that these 
dependencies exist. For instance, for the electric field one should read $\boldsymbol{E}=\boldsymbol{E}(\boldsymbol{r}, t)$, etc.

We suppose the material under consideration is non-magnetic, so that $\boldsymbol{B}=\mu_{0} \boldsymbol{H}$. Then it is easy to derive the wave equation, which can be done in terms of either the electric field $\boldsymbol{E}$ or the magnetic $\boldsymbol{H}$. The speed of light in vacuum appears in the wave equation as $c=1 / \sqrt{\mu_{0} \varepsilon_{0}}$.

In terms of the electric field $\boldsymbol{E}$, the wave equation, which must be satisfied by any electromagnetic wave, is

$$
\nabla \times(\nabla \times \boldsymbol{E})+\frac{1}{c^{2}} \frac{\partial^{2} \boldsymbol{E}}{\partial t^{2}}=-\mu_{0} \frac{\partial^{2} \boldsymbol{P}}{\partial t^{2}} .
$$

We assume that $\boldsymbol{P}$ depends linearly on $\boldsymbol{E}$ :

$$
\boldsymbol{P}=\varepsilon_{0} \chi(\boldsymbol{r}) \boldsymbol{E},
$$

where $\chi(r)$ is the position dependent (for photonic crystals: periodically varying) dielectric susceptibility. This leads to the wave equation in terms of the electric field $\boldsymbol{E}$ :

$$
\nabla \times(\nabla \times \boldsymbol{E})=-\frac{1}{c^{2}}[1+\chi(r)] \frac{\partial^{2} \boldsymbol{E}}{\partial t^{2}} .
$$

In terms of the magnetic field $\boldsymbol{H}$, the wave equation, which must be satisfied by any electromagnetic wave, is

$$
\nabla \times[\eta(r) \nabla \times \boldsymbol{H}]=-\frac{1}{c^{2}} \frac{\partial^{2} \boldsymbol{H}}{\partial t^{2}}
$$

where

$$
\eta(r) \equiv \frac{\varepsilon_{0}}{\varepsilon(r)}=\frac{1}{1+\chi(r)} .
$$

Instead of expressing the wave equation in terms of $\boldsymbol{E}$ or $\boldsymbol{H}$, we can also derive the wave equation in terms of the displacement field $\boldsymbol{D}$.

\subsection{Derivation of the plane-wave expansion method}

The dielectric function $\varepsilon(r)$ of a photonic crystal possesses a lattice periodicity, i.e. it is invariant under a translation over a basis vector of the crystal lattice. Therefore it is convenient to express the material properties (the inverse dielectric function $\eta(\boldsymbol{r})$ ) and the relevant electromagnetic-field components (the magnetic field $\boldsymbol{H}(\boldsymbol{r}, t))$ in terms of their Fourier expansions.

Thus, the periodic dielectric structure is modelled by a Fourier series for the inverse dielectric function $\eta(\boldsymbol{r})$ :

$$
\eta(\boldsymbol{r})=\frac{1}{\varepsilon(\boldsymbol{r})}=\sum_{m} \eta_{m} \exp \left(-\mathrm{i} \boldsymbol{g}_{m} \cdot \boldsymbol{r}\right)
$$


where $m$ labels the three-dimensional set of reciprocal-lattice vectors $\boldsymbol{g}_{m}$. In principle, the summation $\sum_{m}$ is carried out overall reciprocal-lattice vectors that exist in reciprocal space. The Fourier coefficients $\eta_{m}$ are given by

$$
\eta_{m}=\frac{1}{\Omega} \int_{\Omega} \eta(\boldsymbol{r}) \exp \left(\mathrm{i} \boldsymbol{g}_{m} \cdot \boldsymbol{r}\right) \mathrm{d} \boldsymbol{r}
$$

where the integration extends over the volume $\Omega$ of one unit cell in the direct lattice. Because of the inversion symmetry possessed by the unit cell and the fact that the dielectric function $\varepsilon(r)$ is assumed real, the Fourier coefficients $\eta_{m}$ are also real.

Also the magnetic field is expressed as a Fourier series, which is known as the Bloch-wave expansion [39]

$$
\boldsymbol{H}(\boldsymbol{r}, t)=\exp (\mathrm{i} \omega t) \sum_{m} \sum_{\boldsymbol{k}} \sum_{\lambda=1}^{3} h_{m}^{\lambda}(\boldsymbol{k}) \hat{\boldsymbol{u}}_{m}^{\lambda} \exp \left(-\mathrm{i} \boldsymbol{k}_{m} \cdot \boldsymbol{r}\right) .
$$

Here, for any label $m,\left\{\hat{\boldsymbol{u}}_{m}^{1}, \hat{\boldsymbol{u}}_{m}^{2}, \hat{\boldsymbol{u}}_{m}^{3}\right\}$ is a right-handed orthonormal basis for Euclidean space, chosen in such a way that $\hat{u}_{m}^{3} \| \boldsymbol{k}_{m}$. Furthermore, $\boldsymbol{k}_{m}=\boldsymbol{k}+\boldsymbol{g}_{m}$, and the summation over $k$ denotes the summation over all wave vectors within the first Brillouin zone. The length of the vector $\boldsymbol{k}_{m}$ will be denoted $k_{m}$. Note that we write a summation over $\boldsymbol{k}$, in the same way as in Refs. [40,41], whereas it would appear to be more correct to write an integration over $\boldsymbol{k}$, because $\boldsymbol{k}$ is a continuous variable throughout the Brillouin zone.

We can show that the $\lambda=3$ components of the magnetic field vanish because $\nabla \cdot \boldsymbol{H}=0$ :

$$
\begin{gathered}
\nabla \cdot \boldsymbol{H}(\boldsymbol{r}, t)=-\mathrm{i} \exp (\mathrm{i} \omega t) \sum_{m} \sum_{\boldsymbol{k}} \sum_{\lambda=1}^{3} h_{m}^{\lambda}(\boldsymbol{k}) \boldsymbol{k}_{m} \cdot \hat{\boldsymbol{u}}_{m}^{\lambda} \exp \left(-\mathrm{i} \boldsymbol{k}_{m} \cdot \boldsymbol{r}\right) \\
=-\mathrm{i} \exp (\mathrm{i} \omega t) \sum_{m} \sum_{k} \sum_{\lambda=1}^{3} h_{m}^{\lambda}(\boldsymbol{k}) k_{m} \hat{\boldsymbol{u}}_{m}^{3} \cdot \hat{\boldsymbol{u}}_{m}^{\lambda} \exp \left(-\mathrm{i} \boldsymbol{k}_{m} \cdot \boldsymbol{r}\right) \\
=-\mathrm{i} \exp (\mathrm{i} \omega t) \sum_{m} \sum_{k} h_{m}^{3}(\boldsymbol{k}) k_{m} \exp \left(-\mathrm{i} \boldsymbol{k}_{m} \cdot \boldsymbol{r}\right)=0 .
\end{gathered}
$$

This implies that, for any $m$ and any $k, h_{m}^{3}(\boldsymbol{k})=0$. The Bloch-wave expansion for the magnetic field in Eq. (16) is simplified as

$$
\boldsymbol{H}(\boldsymbol{r}, t)=\exp (\mathrm{i} \omega t) \sum_{m} \sum_{\boldsymbol{k}} \sum_{\lambda=1}^{2} h_{m}^{\lambda}(\boldsymbol{k}) \hat{\boldsymbol{u}}_{m}^{\lambda} \exp \left(-\mathrm{i} \boldsymbol{k}_{m} \cdot \boldsymbol{r}\right)
$$

Substituting these Fourier expansions for $\eta(\boldsymbol{r})$, Eq. (14), and for $\boldsymbol{H}(\boldsymbol{r}, t)$, Eq. (18), into the wave equation (12), then leaving out the time-dependence factor $\exp (\mathrm{i} \omega t)$, we arrive at 


$$
\begin{aligned}
& -\sum_{l} \sum_{m} \sum_{k} \sum_{\lambda=1}^{2} k_{l} k_{m} \eta_{m-l} \hat{\boldsymbol{u}}_{m}^{3} \times\left(\hat{\boldsymbol{u}}_{l}^{3} \times \hat{\boldsymbol{u}}_{l}^{\lambda}\right) h_{l}^{\lambda}(\boldsymbol{k}) \exp \left(-\mathrm{i} \boldsymbol{k}_{m} \cdot \boldsymbol{r}\right) \\
& =(\omega / c)^{2} \sum_{m} \sum_{\boldsymbol{k}} \sum_{\lambda=1}^{2} h_{m}^{\lambda}(\boldsymbol{k}) \hat{\boldsymbol{u}}_{m}^{\lambda} \exp \left(-\mathrm{i} \boldsymbol{k}_{m} \cdot \boldsymbol{r}\right) .
\end{aligned}
$$

As two Fourier series are equal, if and only if their Fourier coefficients are equal, this leads to the following set of equations for every $k$ and $m$ :

$$
-\sum_{l} \sum_{\lambda=1}^{2} k_{l} k_{m} \eta_{m-l} \hat{u}_{m}^{3} \times\left(\hat{u}_{l}^{3} \times \hat{u}_{l}^{\lambda}\right) h_{l}^{\lambda}(\boldsymbol{k})=(\omega / c)^{2} \sum_{\lambda=1}^{2} h_{m}^{\lambda}(\boldsymbol{k}) \hat{\boldsymbol{u}}_{m}^{\lambda} .
$$

Working out the summation over $\lambda$ in the left-hand side of Eq. (20), we obtain

$$
-\sum_{l} k_{l} k_{m} \eta_{m-l}\left[\left(\hat{u}_{m}^{3} \times \hat{u}_{l}^{2}\right) h_{l}^{1}(\boldsymbol{k})-\left(\hat{\boldsymbol{u}}_{m}^{3} \times \hat{\boldsymbol{u}}_{l}^{1}\right) h_{l}^{2}(\boldsymbol{k})\right]=(\omega / c)^{2} \sum_{\lambda=1}^{2} h_{m}^{\lambda}(\boldsymbol{k}) \hat{u}_{m}^{\lambda} .
$$

Taking the inner product of Eq. (21) with $\hat{\boldsymbol{u}}_{m}^{1}$ gives

$$
\sum_{l} k_{l} k_{m} \eta_{m-l}\left[\left(\hat{u}_{m}^{2} \cdot \hat{\boldsymbol{u}}_{l}^{2}\right) h_{l}^{1}(\boldsymbol{k})-\left(\hat{\boldsymbol{u}}_{m}^{2} \cdot \hat{\boldsymbol{u}}_{l}^{1}\right) h_{l}^{2}(\boldsymbol{k})\right]=(\omega / c)^{2} h_{m}^{1}(\boldsymbol{k}) .
$$

Taking the inner product of Eq. (21) with $\hat{\boldsymbol{u}}_{m}^{2}$ gives

$$
\sum_{l} k_{l} k_{m} \eta_{m-l}\left[-\left(\hat{\boldsymbol{u}}_{m}^{1} \cdot \hat{\boldsymbol{u}}_{l}^{2}\right) h_{l}^{1}(\boldsymbol{k})+\left(\hat{\boldsymbol{u}}_{m}^{1} \cdot \hat{\boldsymbol{u}}_{l}^{1}\right) h_{l}^{2}(\boldsymbol{k})\right]=(\omega / c)^{2} h_{m}^{2}(\boldsymbol{k}) .
$$

Then we write Eqs. (22) and (23) as a matrix equation

$$
\sum_{l} k_{l} k_{m} \eta_{m-l}\left[\begin{array}{cc}
\hat{\boldsymbol{u}}_{m}^{2} \cdot \hat{\boldsymbol{u}}_{l}^{2} & -\hat{\boldsymbol{u}}_{m}^{2} \cdot \hat{\boldsymbol{u}}_{l}^{1} \\
-\hat{\boldsymbol{u}}_{m}^{l} \cdot \hat{\boldsymbol{u}}_{l}^{2} & \hat{\boldsymbol{u}}_{m}^{1} \cdot \hat{\boldsymbol{u}}_{l}^{1}
\end{array}\right]\left[\begin{array}{c}
h_{l}^{1}(\boldsymbol{k}) \\
h_{l}^{2}(\boldsymbol{k})
\end{array}\right]=(\omega / c)^{2}\left[\begin{array}{c}
h_{m}^{1}(\boldsymbol{k}) \\
h_{m}^{2}(\boldsymbol{k})
\end{array}\right] \text {. }
$$

In practical cases, a finite number of $N$ reciprocal-lattice vectors is selected and the set of fundamental equations (24) is commonly written as a $2 N \times 2 N$ matrix equation. Then the eigenvalues of the $2 N \times 2 N$ matrix must be identified with $(\omega / c)^{2}$, which immediately gives us the photonic band structure. This $2 N \times 2 N$ matrix $\mathcal{M}$ is constructed in the following way. We regard the matrix $\mathcal{M}$ as an $N \times N$ matrix with elements $\mathcal{M}_{m l}$, where each of the labels $m$ and $l$ corresponds to one of the reciprocal-lattice vectors $\boldsymbol{g}_{m}$ and $\boldsymbol{g}_{l}$ selected. In turn, each element $\mathcal{M}_{m l}$ is a $2 \times 2$ matrix, assigned according to the following prescription:

$$
\mathcal{M}_{m l}=k_{l} k_{m} \eta_{m-l}\left[\begin{array}{cc}
\hat{\boldsymbol{u}}_{m}^{2} \cdot \hat{\boldsymbol{u}}_{l}^{2} & -\hat{\boldsymbol{u}}_{m}^{2} \cdot \hat{\boldsymbol{u}}_{l}^{1} \\
-\hat{\boldsymbol{u}}_{m}^{1} \cdot \hat{\boldsymbol{u}}_{l}^{2} & \hat{\boldsymbol{u}}_{m}^{1} \cdot \hat{\boldsymbol{u}}_{l}^{1}
\end{array}\right] \text {. }
$$

Because of the inversion symmetry mentioned above and the assumption that the index of refraction of the material is a real quantity, the matrix $\mathcal{M}$ is real and symmetric. Its eigenvalues are real-valued quantities, to be identified with $(\omega / c)^{2}$. Our calculations show that the eigenvalues are indeed non-negative. 


\section{Results}

We will demonstrate the application of the above approach for the case of two-dimensional (2D) photonic crystals filling a half space. The pertinent 2D free-space Green tensor $\stackrel{\leftrightarrow}{\Gamma}$ reads

$$
\overleftrightarrow{\Gamma}\left(r, r_{S}^{\prime}, \omega\right)=\frac{1}{4 \mathrm{i}}\left[\overleftrightarrow{\mathcal{I}}+(c / \omega)^{2} \nabla \otimes \nabla\right] H_{0}^{(2)}\left(\omega\left|r-r_{S}^{\prime}\right| / c\right)
$$

where $H_{0}^{(2)}$ is the zeroth-order Hankel function of the second kind. The crystal under consideration has a $2 \mathrm{D}$ periodicity of the dielectric function $\varepsilon(\boldsymbol{r})$ in the $x$ and $y$ directions and is infinitely extended in the $z$ direction, see Fig. 2.

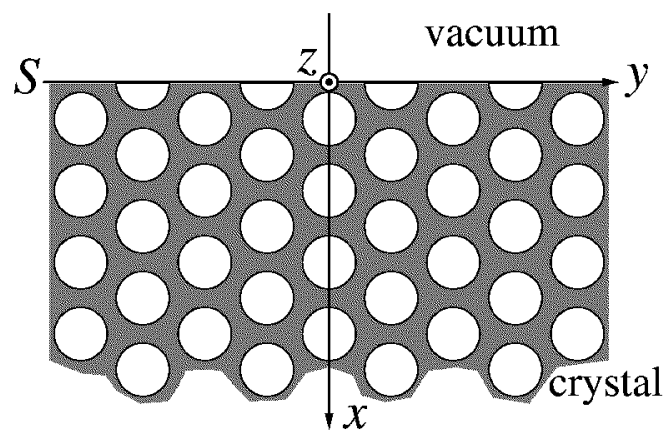

Fig. 2. Cross-section of a two-dimensional photonic crystal with the $x y$-plane. The boundary surface $S$ of the crystal is defined to be the $y z$-plane.

The vector $r$, which occurs in our expressions, is restricted to the $x y$-plane. Since, for purely 2D Maxwell fields there is no mixing between the two polarizations (TE, with the electric field parallel to the $z$-axis, and TM, with the magnetic field parallel to the $z$-axis), we have a set of only two equations for each polarization, namely the continuity of $\boldsymbol{H}_{y}$ and $(\nabla \times \boldsymbol{H})_{z}$ for TE polarization, with the surface currents $\boldsymbol{J}_{1}$ and $\boldsymbol{J}_{2}$ being only in the $y$ direction, and the continuity of $\boldsymbol{H}_{z}$ and $(\nabla \times \boldsymbol{H})_{y}$ for TM polarization, with $\boldsymbol{J}_{1}$ and $\boldsymbol{J}_{2}$ being only in the $z$ direction.

We have used our method to reconstruct the reflected fields in the vacuum region from the surface-current distribution $\boldsymbol{J}_{1}$. In particular, we have calculated the distribution of the energy flow over all outgoing directions $\alpha$ (measured with respect to the surface normal), upon irradiation of the crystal by a plane wave. For this intensity distribution we use the asymptotic behaviour of $\stackrel{\leftrightarrow}{\Gamma}$ at large distances away from the crystal surface. As a measure for the intensity we use the dimensionless quantity $I(\alpha) \equiv D|\boldsymbol{H}|^{2} /\left|\boldsymbol{H}_{\text {in }}\right|^{2}$, where $D$ is the ratio of the distance at which the field is observed and the length of the crystal surface. This measure is independent of $D$, as for $2 \mathrm{D}$ systems $|\boldsymbol{H}|$ is inversely proportional to $\sqrt{D}$.

As a first test of the numerical implementation of our method, we have considered the limiting case of a homogeneous medium $(\varepsilon(r)=\varepsilon=$ const) filling 
the half space $x>0$. The Green tensor for the homogeneous medium is given by that of the vacuum (Eq. (26)), with $c$ replaced by $c / \sqrt{\varepsilon}$. For all angles of incidence considered, we have found that the numerically obtained angular distribution of the reflected and the transmitted intensity, agrees perfectly with the theoretically expected behaviour based upon Fresnel's and Snell's laws. In particular, for TM polarised light incident under Brewster's angle $\theta_{\mathbf{B}}=\arctan \sqrt{\varepsilon}$, the intensity of the reflected light vanishes, both theoretically and in our numerical results.

Next, we have considered the scattering of a plane wave incident on a $2 \mathrm{D}$ photonic crystal consisting of air cylinders (radius $R=0.48 a$ ), arranged on a triangular lattice (lattice constant $a$ ) in a dielectric background. The orientation of the lattice is as shown in Fig. 2. We will present results for two particular configurations of the crystal, which only differ in the value of the dielectric contrast.

Figure 3 shows the angular distribution of the reflected intensity in the case of a weakly scattering crystal where the dielectric background has index of refraction $\sqrt{\varepsilon}=1.05$, i.e., a weakly scattering crystal. We considered a scattering surface with length $L=40 a$, which was discretised into 401 points. The calculation of the Green tensor of the crystal was based on the plane-wave expansion method using 283 plane waves. The integration over the first Brillouin zone in Eq. (4) was performed by discretising half of this BZ into 12286 points. For the absorption rate we used $\gamma=0.005 \omega$. The incident plane wave was taken TM polarised and incident normally to the surface, its frequency ranging from $\omega=4.071 \mathrm{c} / \mathrm{a}$ to $\omega=4.531 \mathrm{c} / \mathrm{a}$.

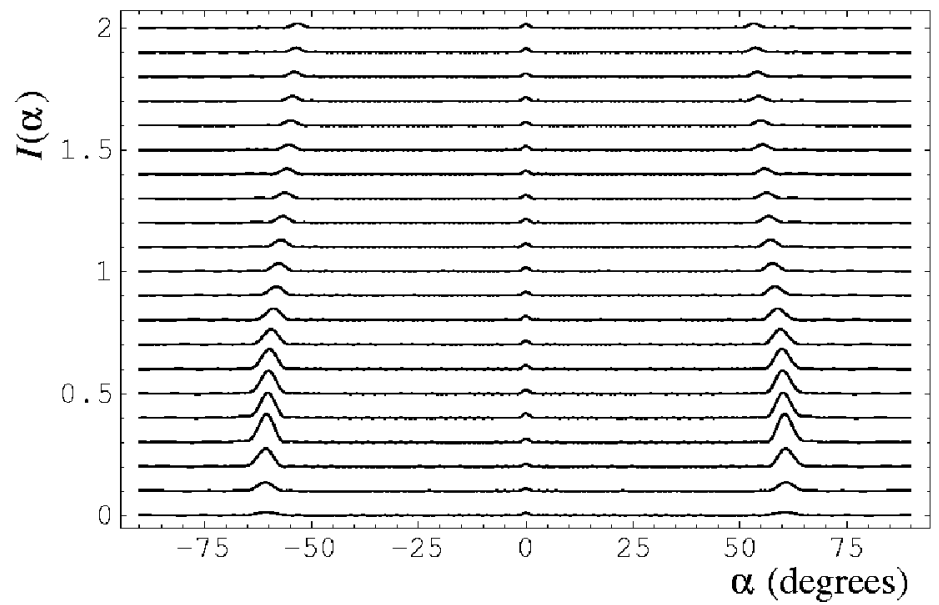

Fig. 3. Angular distribution of the intensity of the light reflected from a weakly scattering $2 \mathrm{D}$ photonic crystal consisting of air cylinders (radius $R=0.48 a$ ), arranged on a triangular lattice (lattice constant $a$ ) in a dielectric background (index of refraction $\sqrt{\varepsilon}=1.05$ ). The incident light consists of a TM polarised plane wave, incident normally onto the scattering surface, with frequency ranging from $\omega=4.071 \mathrm{c} / \mathrm{a}$ (bottom curve, no offset) to $\omega=4.531 \mathrm{c} / \mathrm{a}$ (top curve, offset 2.0 ), using steps of $\Delta \omega=0.023 \mathrm{c} / \mathrm{a}$. 
In Fig. 3, we observe that part of the light is reflected normal to the crystal surface. This is the specularly reflected part of the field, which for the present weak contrast is very small. In addition to this specular reflection, we observe two peaks, occurring at $\alpha \approx \pm 60^{\circ}$. These are the Bragg peaks, which follow from single scattering of the incident beam on the dielectric periodicity (first-order Born's approximation). Momentum conservation dictates that the wave vector of the outgoing beam differs from the incident one by a reciprocal lattice vector. If we also account for energy conservation, it turns out that for the present geometry Bragg scattering ideally should only occur for $\omega=\omega_{0}=(4 \pi / 3) c / a \approx 4.189 \mathrm{c} / a$, with scattering angles $\alpha= \pm 60^{\circ}$. This ideal situation is represented by the sixth curve from below in Fig. 3 . The finite width of both peaks results from the finite length of the crystal surface $L=40 a$.

We observe, however, that for a range of frequencies around the resonance frequency $\omega_{0}$, the Bragg peaks survive. This is due to the finite damping rate $\gamma$, which may be interpreted as leading to a finite depth of the crystal, thus breaking momentum conservation in the $x$ direction. A simple calculation, based on the conservation of momentum in the $y$ direction (the direction parallel to the crystal surface), shows that the angle $\alpha$ must satisfy the relation $\alpha=\arcsin \left(\frac{1}{2} \sqrt{3} \omega_{0} / \omega\right)$. This explains accurately the shifting of the peak position in Fig. 3 from $\alpha= \pm 61^{\circ}$ at $\omega=4.071 \mathrm{c} / \mathrm{a}$ (bottom curve) to $\alpha= \pm 53^{\circ}$ at $\omega=4.531 \mathrm{c} / \mathrm{a}$ (top curve). The width of the frequency region in which the Bragg peaks can be observed agrees well with estimates based on the finite effective crystal depth imposed by $\gamma$. We finally note that changing the index of refraction to $\sqrt{\varepsilon}=1.1$ leaves the angular intensity distributions identical, except for changing the vertical scale by a factor of 4 , which confirms that we are in the single-scattering regime.

We have repeated these calculations for the same crystal geometry, but now with a dielectric background of $\sqrt{\varepsilon}=4.0$. For this strong-scattering case, a full band gap develops for both TM and TE polarization, as is clearly seen in the calculated band structure plotted in Fig. 4a. The angular distribution of the reflected TM light for this crystal is given in Fig. $4 \mathrm{~b}$ for a range of frequencies, all lying above the band gap (frequencies inside the gap give rise to complete specular reflection, as there are no Bloch modes to couple the light to).

Again, we observe the specular reflection and the "Bragg" peaks, which, of course, all have a strongly increased intensity compared to the weak-scattering case. More interesting is the observation that the range of frequencies over which the "Bragg" peaks are observed has grown appreciably compared to the case of weak scattering. While we are unable to give a simple theoretical estimate of this frequency range, like we did for the case of weak scattering, it is noteworthy that over the entire range the position of the peak is again accurately described by $\alpha=\arcsin \left(\frac{1}{2} \sqrt{3} \omega_{0} / \omega\right)$. The frequency for which the thus calculated angle becomes $\alpha= \pm 90^{\circ}$, equals $(2 \pi / \sqrt{3}) c / a$, which lies just above the stop gap in the $K$ direction (direction of incidence) and agrees with the second curve from below in Fig. 4b. 


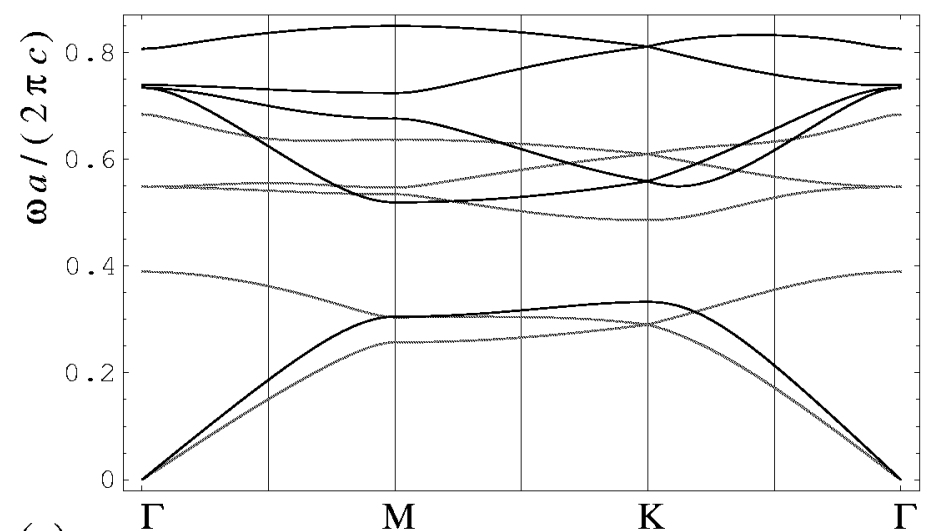

(a)

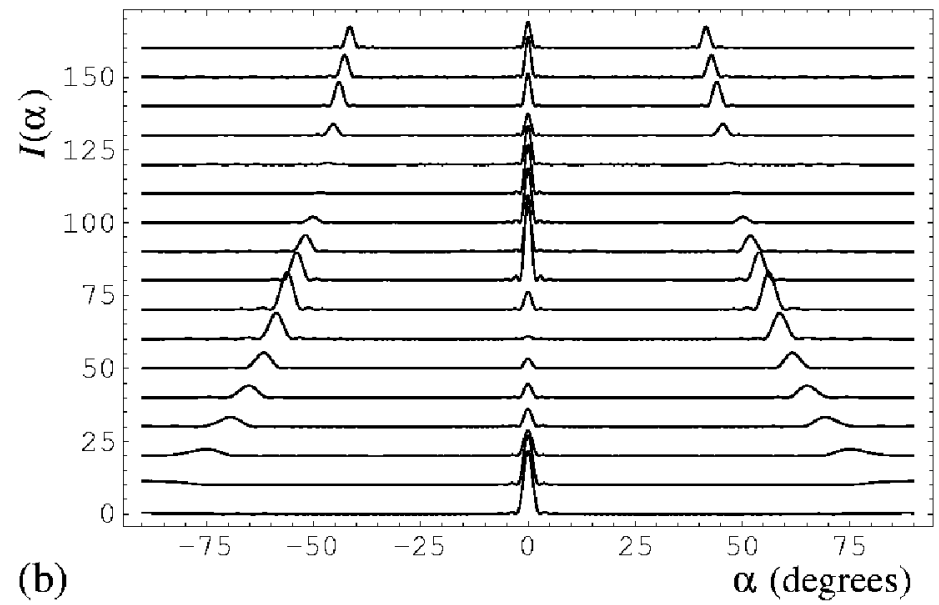

Fig. 4. (a) Band structure for the strongly scattering 2D photonic crystal with the same parameters as in Fig. 3, except that the background index of refraction is $\sqrt{\varepsilon}=4.0$. The black (grey) curves correspond to the TM (TE) modes. (b) Angular distribution of the intensity of the light reflected from this $2 \mathrm{D}$ photonic crystal, upon irradiation by a TM plane wave of normal incidence, with frequencies ranging from $\omega=3.512 c / a$ (bottom curve, no offset) to $\omega=5.464 c / a$ (top curve, offset 160 ), using steps of $\Delta \omega=0.122 c / a$.

We also note an interesting nonmonotonic behaviour of the intensities of the peaks as a function of the frequency, where it seems that a redistribution of energy takes place between the specular and the "Bragg" peaks. It will be of interest to monitor this behaviour in experiments on strongly scattering crystals with a small but finite damping rate.

\section{Discussion}

We have presented a method to calculate the scattering of light at the sur- 
face of a photonic crystal. The scattering problem is solved in terms of virtual surface-current distributions and the calculation takes full advantage of the existing infinite-space plane-wave expansion method for obtaining the photonic band structure. Working with surface currents reduces the dimensionality in the problem and thus also reduces the required computer time and memory. We have tested our method on the cases of scattering at the surfaces of a homogeneous medium and a weakly scattering $2 \mathrm{D}$ photonic crystal; all results were found to agree accurately with theoretical expectations. We have also used the method for a first study on a strongly scattering crystal, where simple arguments based on Born's approximation break down. A more extensive analysis of strongly scattering crystals, as well as the application of our method to more general situations (crystals of different shapes and incident light of non-plane-wave nature) will be the topic of further study.

\section{References}

[1] V.P. Bykov, Sov. J. Quantum Electron. 4, 861 (1975).

[2] E. Yablonovitch, Phys. Rev. Lett. 58, 2059 (1987).

[3] S. John, Phys. Rev. Lett. 58, 2486 (1987).

[4] J.D. Joannopoulos, R.D. Meade, J.N. Winn, Photonic Crystals: Molding the Flow of Light, Princeton University Press, Princeton 1995.

[5] J.D. Joannopoulos, P.R. Villeneuve, S. Fan, Solid State Commun. 102, 165 (1997).

[6] H.M. van Driel, W.L. Vos, Phys. Rev. B 62, 9872 (2000).

[7] S. Enoch, G. Tayeb, D. Maystre, Opt. Commun. 161, 171 (1999).

[8] S. John, T. Quang, Phys. Rev. A 50, 1764 (1994).

[9] M. Megens, J.E.G.J. Wijnhoven, A. Lagendijk, W.L. Vos, Phys. Rev. A 59, 4727 (1999).

[10] M. Megens, J.E.G.J. Wijnhoven, A. Lagendijk, W.L. Vos, J. Opt. Soc. Am. B 16, 1403 (1999).

[11] G. Kurizki, A.Z. Genack, Phys. Rev. Lett. 61, 2269 (1988).

[12] E. Yablonovitch, J. Opt. Soc. Am. B 10, 283 (1993).

[13] E. Yablonovitch, J. Phys., Condens. Matter 5, 2443 (1993).

[14] M. Bayindir, B. Temelkuran, E. Özbay, Phys. Rev. B 61, 11855 (2000).

[15] A. Mekis, J.C. Chen, I. Kurland, S. Fan, P.R. Villeneuve, J.D. Joannopoulos, Phys. Rev. Lett. 77, 3787 (1996).

[16] S.Y. Lin, E. Chow, V. Hietala, P.R. Villeneuve, J.D. Joannopoulos, Science 282, 274 (1998).

[17] M. Tokushima, H. Kosaka, A. Tomita, H. Yamada, Appl. Phys. Lett. 76, 952 (2000).

[18] M. Bayindir, E. Özbay, B. Temelkuran, M.M. Sigalas, C.M. Soukoulis, R. Biswas, K.M. Ho, Phys. Rev. B 63, 081107 (2001). 
[19] M. Bayindir, B. Temelkuran, E. Özbay, Appl. Phys. Lett. 77, 3902 (2000).

[20] S. Fan, S.G. Johnson, J.D. Joannopoulos, C. Manolatou, H.A. Haus, J. Opt. Soc. Am. B 18, 162 (2001).

[21] S.G. Johnson, P.R. Villeneuve, S. Fan, J.D. Joannopoulos, Phys. Rev. B 62, 8212 (2000).

[22] D. Labilloy, H. Benisty, C. Weisbuch, T.F. Krauss, R. Houdré, U. Oesterle, Appl. Phys. Lett. 71, 738 (1997).

[23] C.J.M. Smith, H. Benisty, S. Olivier, M. Rattier, C. Weisbuch, T.F. Krauss, R.M. de la Rue, R. Houdré, U. Oesterle, Appl. Phys. Lett. 77, 2813 (2000).

[24] O. Painter, R.K. Lee, A. Scherer, A. Yariv, J.D. O'Brien, P.D. Dapkus, I. Kim, Science 284, 1819 (1999).

[25] C.J.M. Smith, T.F. Krauss, H. Benisty, M. Rattier, C. Weisbuch, U. Oesterle, R. Houdré, J. Opt. Soc. Am. B 17, 2043 (2000).

[26] E.R. Brown, C.D. Parker, E. Yablonovitch, J. Opt. Soc. Am. B 10, 404 (1993).

[27] S.D. Cheng, R. Biswas, E. Özbay, J.S. McCalmont, G. Tuttle, K.M. Ho, Appl. Phys. Lett. 67, 3399 (1995).

[28] M. Doosje, B.J. Hoenders, J. Knoester, J. Opt. Soc. Am. B 17, 600 (2000).

[29] K.M. Ho, C.T. Chan, C.M. Soukoulis, Phys. Rev. Lett. 65, 3152 (1990).

[30] P.R. Villeneuve, M. Piché, J. Mod. Opt. 41, 241 (1994).

[31] R. Biswas, M.M. Sigalas, G. Subramania, K.M. Ho, Phys. Rev. B 57, 3701 (1998).

[32] K. Busch, S. John, Phys. Rev. E 58, 3896 (1998).

[33] O.J.F. Martin, N.B. Piller, Phys. Rev. E 58, 3909 (1998).

[34] M. Sigalas, C.M. Soukoulis, E.N. Economou, C.T. Chan, K.M. Ho, Phys. Rev. B 48, 14121 (1993).

[35] J.B. Pendry, J. Mod. Opt. 41, 209 (1994).

[36] G. Lauricella, Nuovo Cimento 13, 254 (1907).

[37] K. Sakoda, K. Ohtaka, Phys. Rev. B 54, 5732 (1996).

[38] J.A. Stratton, Electromagnetic Theory, McGraw-Hill, New York 1941, Sec. 1.13.

[39] N.W. Ashcroft, N.D. Mermin, Solid State Physics, Holt, Rinehart, and Winston, Austin 1976.

[40] K.W.K. Shung, Y.C. Tsai, Phys. Rev. B 48, 11265 (1993).

[41] İ.I. Tarhan, G.H. Watson, Phys. Rev. B 54, 7593 (1996). 